\title{
ABUNDANCE ANOMALIES IN GLOBULAR CLUSTERS
}

\author{
G. S. DA COSTA \\ Mt. Stromlo $\mathcal{E}$ Siding Spring Observatories, \\ Private Bag, Weston Creek PO, ACT 2611, AUSTRALIA
}

\section{Introduction}

The galactic globular clusters presumably formed rapidly as high density concentrations at the centres of extensive star forming regions and the natural expectation is that they will be chemically homogeneous. In general, this is confirmed by observation - most globular clusters are extremely homogeneous with regard to most elements. (There are two exceptions: $\omega$ Cen and M22 both have intrinsic internal abundance ranges. While of considerable interest, e.g. Norris et al. 1996, this type of "abundance anomaly" will not be discussed here). But we have known for more than twenty years ${ }^{1}$ that the surface abundances, which are what we observe, of elements such as $\mathrm{C}, \mathrm{N}$ and $\mathrm{O}$ can vary substantially from red giant to red giant within an individual globular cluster. Indeed it has become clear that "abundance anomalies" of this type are common in the galactic globular cluster population. Briefly, the observed anomalies can be summarized as: (i) the "anomalous" stars are depleted in $\mathrm{C}$ and enhanced in N. Depletions of $\mathrm{O}$ also often accompany the depletions in $\mathrm{C}$. (ii) The $\mathrm{C}, \mathrm{N}$ and $\mathrm{O}$ variations are usually accompanied by enhancements of $\mathrm{Na}$ and $\mathrm{Al}$ and when the $\mathrm{O}$ depletion and the $\mathrm{Al}$ enhancement are both large, $\mathrm{Mg}$ is depleted. No other elements, including $\mathrm{r}$ - and s-process indicators, vary.

There are two basic competing explanations for these observations, though it is quite possible that both processes occur to varying extents in most clusters. The first is the Evolutionary Hypothesis in which the abundance anomalies are the result of internal processes. An example would be the mixing of nuclear processed material from the interior into the surface layers. The second explanation is the Primordial Hypothesis in which the

\footnotetext{
${ }^{1}$ The origin of this subject is often ascribed to the DDO photometry of Osborn (1971) and Hesser et al. (1976). However, Harding (1962) had discovered a CH-star in $\omega$ Cen, and even earlier, Popper (1947) noted that the M13 red giant L199 had strong CN bands when compared to other giants in the cluster. Modern work has confirmed L199 as a CN-strong star.
} 


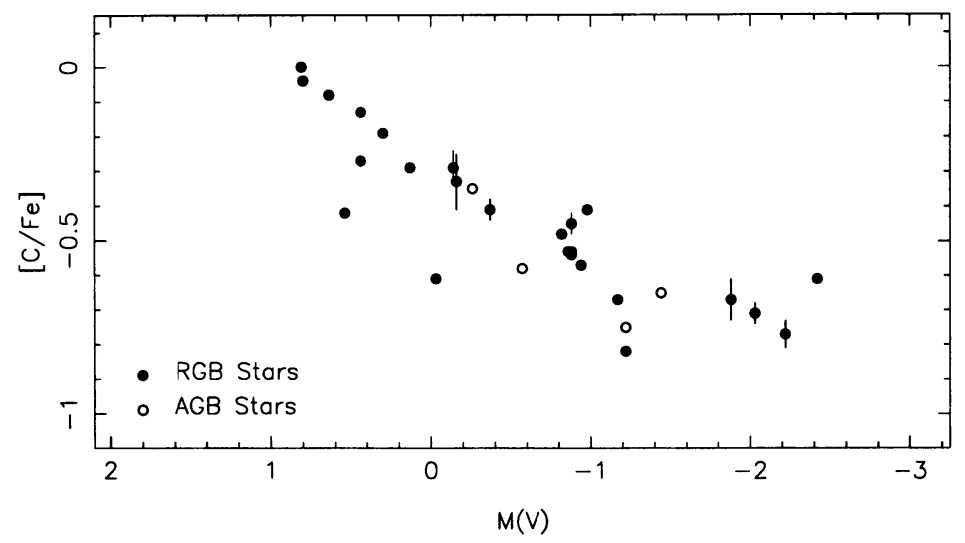

Figure 1. Carbon abundances on the giant branch as a function of luminosity in the metal-poor globular cluster NGC 6397. Data from Briley et al. (1990).

abundance anomalies result from external processes, most probably at the time the cluster formed. For example, if the proto-cluster gas cloud was not well-mixed chemically, then the stars that form from it would not have identical compositions.

\section{Metal-Poor Clusters}

\subsection{THE OBSERVATIONS}

There are a number of galactic globular clusters with $[\mathrm{Fe} / \mathrm{H}] \leq-1.0$ for which spectroscopic observations of large samples of cluster members exist. From these datasets a number of general results for the $\mathrm{C}, \mathrm{N}$ and $\mathrm{O}$ abundances can be inferred. (i) In those clusters where there are data over a sufficiently large luminosity interval, the $\mathrm{C}$ abundance increases, or more correctly, is less depleted, with decreasing luminosity. This is illustrated in Fig. 1 where the results of Briley et al. (1990) for the cluster NGC 6397 are shown. Similar trends of smaller $\mathrm{C}$ depletions at lower luminosities are also seen in the clusters M4 and NGC 6752 (Suntzeff \& Smith 1991) and M92 (Langer et al. 1986). In M92 in particular, the lowest luminosity stars with $\mathrm{C}$ abundance determinations are almost at the main sequence turnoff, and at this luminosity, the abundance ratio $[\mathrm{C} / \mathrm{Fe}]$ is comparable to that for field subdwarfs of similar $[\mathrm{Fe} / \mathrm{H}]$. Further, in all these clusters, at a fixed luminosity there is a range in the observed $\mathrm{C}$ abundance that is not due to observational error only; an intrinsic range in the $\mathrm{C}$ depletions is present. (ii) The depletion of $\mathrm{C}$ is accompanied by an enhancement in N. (iii) $\mathrm{A}$ range of $\mathrm{O}$ depletions of up to 1 dex is seen in many clusters (e.g. Kraft et al. 1995 and references therein). More specifically, where such data exist, the $\mathrm{O}$ and $\mathrm{C}$ depletions are tightly coupled (e.g. Dickens et al. 1991, Smith et al. 1996). (iv) As demonstrated by, for example, Smith et al. (1996) for 


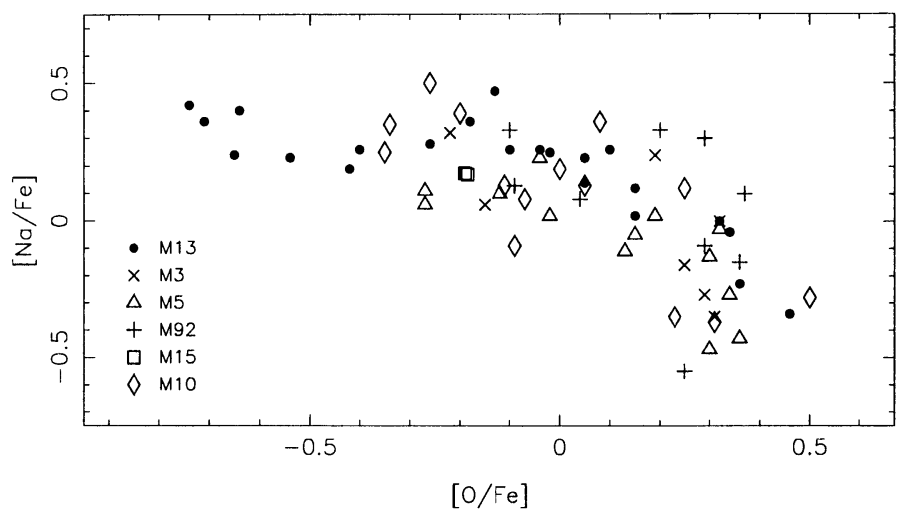

Figure 2. The relationship between $\mathrm{Na}$ enhancement and $\mathrm{O}$ depletion for bright red giants in six globular clusters (see Kraft et al. 1995 and references therein).

M13 and M3, and Dickens et al. (1991) for NGC 362 and NGC 288, the total $\mathrm{C}+\mathrm{N}+\mathrm{O}$ abundance for the red giants in a cluster is generally constant, despite the large ranges seen in the individual $\mathrm{C}, \mathrm{N}$ and $\mathrm{O}$ abundances.

This well-documented change in abundance with evolutionary state is incompatible with any primordial process and so can only be explained as an evolutionary process. The constancy of the $\mathrm{C}+\mathrm{N}+\mathrm{O}$ abundance strongly suggests that we are seeing the mixing of material that has been processed through the $\mathrm{CN}$-cycle (in which $\mathrm{C}$ is converted to $\mathrm{N}$ ) and the $\mathrm{ON}$-cycle (in which $\mathrm{O}$ is converted to $\mathrm{N}$ ) from the interior of the star into its outer layers.

Are the $\mathrm{C}, \mathrm{N}$ and $\mathrm{O}$ abundance variations correlated with abundance anomalies in any other elements? Cottrell \& Da Costa (1981) and Norris et al. (1981) were the first to notice that in the cluster NGC 6752, the Cdepleted, $\mathrm{N}$-enhanced stars also possess enhanced $\mathrm{Na}$ and $\mathrm{Al}$ abundances relative to the $\mathrm{C}$ - and N-normal red giants, while Peterson (1980) noted an apparent connection between $\mathrm{Na}$ enhancements and $\mathrm{O}$ depletions for red giants in M13. These results have now been extended to many clusters and there is a well established anti-correlation between the size of the $\mathrm{Na}$ and $\mathrm{Al}$ enhancements and the degree of $\mathrm{O}$ depletion. The relation for $\mathrm{Na}$ is shown in Fig. 2 while the equivalent relation for $\mathrm{Al}$ is shown in Norris \& Da Costa (1995a) or Shetrone (1996a, hereafter S96a).

Most of these results are derived from spectra of the brightest giants in each cluster. Pilachowski et al. (1996) and Kraft et al. (1997, hereafter K97), however, have extended the observations in M13 to luminosities almost as faint as the horizontal branch. These observations reveal that the relations seen among the brighter red giants are still prevalent at fainter luminosities. These studies also provide information on $\mathrm{Mg}$ abundance variations in M13 (see also S96a). In particular, K97 show that in those M13 stars that have large $\mathrm{Al}$ enhancements and large $\mathrm{O}$ depletions, the $\mathrm{Mg}$ abundance is de- 
pleted by as much as 0.3 dex. A similar $\mathrm{Mg}$ - $\mathrm{Al}$ abundance anti-correlation is present in the $\omega$ Cen data of Norris \& Da Costa (1995b). It is also seen, though to a lesser degree, in the M5 and M92 bright red giant data of S96a. Intriguingly, $\mathrm{K} 97$ also show that in $\mathrm{M} 13$, the total $\mathrm{Mg}+\mathrm{Al}$ abundance is approximately constant, regardless of the degree of $\mathrm{Mg}$ depletion and $\mathrm{Al}$ enhancement (see also S96a). This strongly suggests that the additional $\mathrm{Al}$ atoms present in the $\mathrm{O}$ depleted stars are produced at the expense of $\mathrm{Mg}$ atoms in the same star, a situation reminiscent of the constancy of total $\mathrm{C}+\mathrm{N}+\mathrm{O}$ despite large variations in the individual abundances. It is also significant that the $\mathrm{Na}$ and $\mathrm{Al}$ enhancements and associated $\mathrm{O}$ and $\mathrm{Mg}$ depletions are of size comparable to those seen near the RGB tip, even at the faintest luminosities $\left(\mathrm{M}_{\mathrm{V}} \approx+0.3\right)$ studied by K97.

\subsection{DOES STELLAR EVOLUTION THEORY OFFER AN EXPLANATION?}

The simple answer to the question posed in the section title is "No" standard stellar evolution sequences for red giants do not predict any of the observations described in the previous section. At most, they predict a modest $\mathrm{C}$ depletion and $\mathrm{N}$ enhancement as a result of "first dredge-up" at relatively bright luminosities on the red giant branch. Nevertheless, we can use stellar evolution to provide guidance in interpreting the observations. For example, as first noted by Denisenkov \& Denisenkova (1990), in the region of the H-burning shell in the interior of a red giant, conditions are such that proton capture reactions on $\mathrm{Ne}$ and $\mathrm{Mg}$ seeds can generate $\mathrm{Na}$ and $\mathrm{Al}$ abundance enhancements. This possibility has been explored in more detail recently - see Cavallo et al. (1996) and the references therein. Of course the region where these reactions occur is also that where $\mathrm{O}$ and $\mathrm{C}$ are depleted and $\mathrm{N}$ is enhanced. Consequently, at least in principle, the observations can be explained by mixing the processing products from the vicinity of the H-burning shell into the surface layers.

There are, however, some quantitative problems with this scenario. First, observationally, in the $\mathrm{O}$-depleted stars the $\mathrm{Al}$ enhancement is as large as, if not larger, than the $\mathrm{Na}$ enhancement. But in the nucleosynthesis calculations, generally more $\mathrm{Na}$ than $\mathrm{Al}$ is produced, except perhaps near the RGB tip (Cavallo et al. 1996). Second, in the theoretical calculations, there is little change in the total $\mathrm{Mg}$ abundance (which is dominated by the abundance of ${ }^{24} \mathrm{Mg}$ ) and the $\mathrm{Al}$ enhancement is produced principally from ${ }^{25,26} \mathrm{Mg}$. Yet, at least in M13, the observations show that in many of the stars with large $\mathrm{O}$ depletions and $\mathrm{Al}$ enhancements, the (total) $\mathrm{Mg}$ abundance is depleted by a factor of $\sim 2$ (K97), in disagreement with the theoretical calculations.

It is possible that some of these discrepancies could be removed by changes in the reaction rates used in the calculations, since, for example, Arnould et al. (1995) have shown that the rates of some of the reactions in 
the $\mathrm{NaNe}$ and $\mathrm{AlMg}$ cycles are uncertain by orders of magnitude. Indeed this may be a situation where observations will serve as a guide for theory. However, it is also possible that the discrepancy with the observations may be revealing a more significant problem in the standard evolutionary models. For example, Arnould et al. (1995) show that significant depletions in ${ }^{24} \mathrm{Mg}$ occur in the AlMg cycle only for temperatures above $\sim 70$ million degrees. But such temperatures do not occur in the H-burning shells of standard red giant models. The observation of $\mathrm{Mg}$ (and $\mathrm{O}$ ) depletion and $\mathrm{Al}$ enhancement at relatively low luminosities on the red giant branch in M13 (K97) is particularly worrisome in this regard since, at least in standard models, the H-burning shell in such stars has a temperature well below 70 million degrees. Further, the work of Shetrone (1996b) has demonstrated that we cannot appeal to unusual $\mathrm{Mg}$ isotopic ratios as the explanation. His observations of a small sample of bright red giants in M13 demonstrate unequivocally that the $\mathrm{Mg}$ depletions in the $\mathrm{Al}$ enhanced stars are due to depletions in ${ }^{24} \mathrm{Mg}$. The ${ }^{25} \mathrm{Mg}$ and ${ }^{26} \mathrm{Mg}$ abundances do not vary in any significant way with the degree of $\mathrm{Al}$ enhancement (Shetrone 1996b).

\subsection{OTHER CONSTRAINTS}

There are at least three other issues that should be kept in mind when considering the origin of these abundance anomalies. First, it is extremely unlikely that any large scale mixing process operates on the main sequence in these clusters, for the following reason. Any mixing out of processed material to the surface layers of the star inevitably requires mixing in of fresh hydrogen, and this modifies the star's evolution significantly (e.g. VandenBerg \& Smith 1988). Thus a hypothesized stochastical distribution of mixing extents on the main sequence in a cluster would not generate the tight narrow cluster main sequence turnoff loci that are, in fact, observed. The observations of the $\mathrm{C}$ abundance decline with evolutionary state in clusters such as M92 are not incompatible with this requirement, as long as the mixing begins after the turnoff (VandenBerg \& Smith 1988) as appears to be the case (Langer et al. 1986). However, Suntzeff \& Smith (1991) claim the existence of anti-correlated $\mathrm{CN}$ and $\mathrm{CH}$ band strength variations on the main sequence (at $\mathrm{M}_{\mathrm{V}} \approx+6$ ) in NGC 6752 . If (when) verified by larger samples and spectra with higher $\mathrm{S} / \mathrm{N}$, these observations will be very difficult to interpret in terms of any evolutionary mixing hypothesis.

Second, a "bump" in the luminosity function for the red giant branch is seen in many clusters, including clusters such as M5, M3 and NGC 6752 which show significant abundance anomalies. This feature, which results from the slow down in RGB evolution when the H-burning shell reaches the composition discontinuity left behind by the earlier deepest penetration of the convective envelope, occurs at approximately the luminosity predicted by standard evolutionary models. The existence of this bump indicates that 
not all stars in a cluster can have mixed the region between the H-burning shell and the surface at relatively low luminosities on the red giant branch, else the luminosity function feature would not be detected.

Finally, it is vital to remember that the abundance anomalies discussed here are confined primarily to globular cluster stars. Field stars show these anomalies only to a much smaller extent (e.g. Fig. 8 of Pilachowski et al. 1996). Hence any explanation of the globular cluster star abundance anomalies must also be capable of accommodating this striking cluster star/field star difference.

\section{47 Tucanae - A Special Case?}

The comparatively metal-rich $([\mathrm{Fe} / \mathrm{H}]=-0.7)$ globular cluster 47 Tuc has been the subject of a number of intensive studies. Norris \& Freeman (1979) were the first to show that the distribution of strengths of the $\lambda 4216 \AA$ $\mathrm{CN}$-band in the spectra of cluster red giants was bimodal - stars could be classified as "CN-strong" or "CN-weak". Further, this bimodality is also present on the horizontal branch (Norris \& Freeman 1982) and on the asymptotic giant branch (Norris \& Freeman 1979). In these data there are no indications that the $\mathrm{CN}$-strong to $\mathrm{CN}$-weak number ratio is any different between the red giant, horizontal and asymptotic giant branches, i.e. there is no support for any change in this ratio with evolutionary state, as might have been expected in an evolutionary mixing scenario. However, as demonstrated in Norris et al. (1984), there is a general anti-correlation between the $\mathrm{CN}$ band strengths and the strength of the $\mathrm{G}$ band $(\mathrm{CH})$ $\mathrm{CN}$-strong stars have weaker $\mathrm{CH}$ features (and vice versa) consistent with the involvement of the $\mathrm{CN}$-cycle.

Norris \& Freeman (1979) and later Paltoglou (1989) have also shown that among the red giants, there is a radial gradient in the CN-strong to $\mathrm{CN}$-weak number ratio in the sense that $\mathrm{CN}$-strong stars are considerably more common in the inner regions of the cluster. Figure 3 shows data from Paltoglou (1989) which reveals a decline in the number ratio with radius out to $\sim 10$ arcmin, beyond which the ratio appears to remain approximately constant. Aside from $\omega$ Cen where there is a distinct radial abundance gradient (e.g. Norris et al. 1996), no other globular cluster shows evidence for a similar radial gradient.

As regards other elements, Cottrell \& Da Costa (1981) first showed that there are $\mathrm{Na}$ abundance variations which correlate positively with $\mathrm{CN}$ strength in 47 Tuc red giants. Subsequent work (e.g. Brown \& Wallerstein 1992) has confirmed a $\mathrm{Na}$ abundance difference of $\sim 0.3$ dex between the two groups of giants, though the sample studied at high dispersion remains small. In contrast to the more metal-poor globular clusters, however, there is no indication within this small sample of any $\mathrm{Al}, \mathrm{Mg}$ or $\mathrm{O}$ abundance differences between the $\mathrm{CN}$-strong and $\mathrm{CN}$-weak stars (Cottrell \& Da Costa 


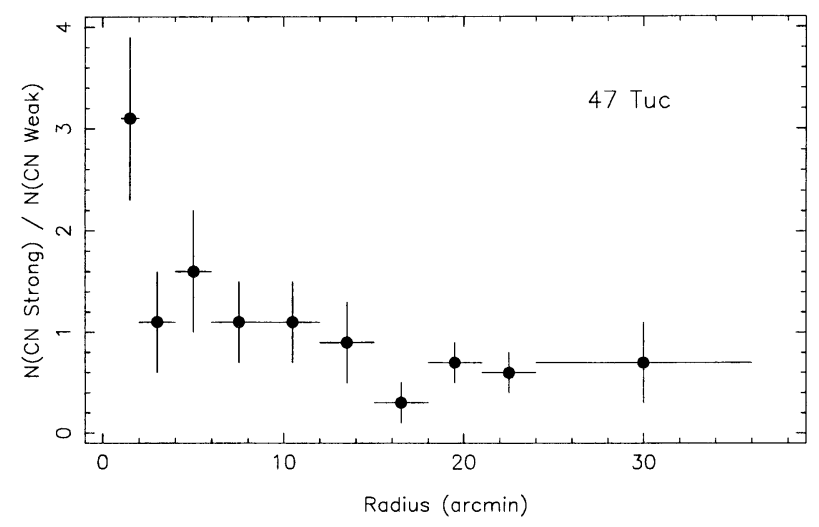

Figure 3. Ratio of the number of $\mathrm{CN}$-strong red giants to the number of $\mathrm{CN}$-weak red giants in 47 Tuc as a function of distance from the cluster centre. There are $\sim 30$ to 40 stars per radial bin; data from Paltoglou (1989).

1981, Brown et al. 1990, Brown \& Wallerstein 1992). It appears that in this cluster the abundance anomalies are restricted to $\mathrm{C}, \mathrm{N}$ and $\mathrm{Na}$ only.

There is one further characteristic that distinguishes 47 Tuc from other globular clusters and that is the availability of reasonable quality spectra for a modest sample of stars at the cluster main sequence turnoff (Briley et al. 1994). The results for this sample of stars are shown in Fig. 4. It is clear from the upper panel of Fig. 4 that there is also a bimodal distribution of $\mathrm{CN}$-band strengths among these main sequence turnoff stars - they can be classified "CN-strong" or "CN-weak" in the same way as the more luminous red giants. Indeed to within the uncertainties inherent in this limited sample, the $\mathrm{CN}$-strong to $\mathrm{CN}$-weak number ratio for these main sequence turnoff stars is the same as it is at higher luminosities. In other words, again there is no evidence for a change in the $\mathrm{CN}$-strong to $\mathrm{CN}$-weak number ratio with evolutionary state. In the lower panel of Fig. 4 it is also apparent that there is a general anti-correlation between the $\mathrm{CN}$ and $\mathrm{CH}$-band strengths, just as is found at higher luminosities. The existence of a $\mathrm{CH} / \mathrm{CN}$ anti-correlation is usually regarded as a signature of a mixing process but for these main sequence turnoff stars, as noted in the previous section, it is difficult to invoke a mixing mechanism to explain the abundance differences.

In a further paper, Briley et al. (1996) present observations at the $\mathrm{Na} D$ lines of a subset of the 47 Tuc main sequence turnoff stars shown in Fig. 4. Significantly, the three CN-strong stars observed have, on average, $\mathrm{Na}$ $\mathrm{D}$ lines that are $\sim 30 \%$ stronger than for three $\mathrm{CN}$-weak stars. In other words, there is evidence for a positive sodium-cyanogen correlation at the main sequence turnoff in 47 Tuc. In addition, the Na abundance difference between the two groups of stars is comparable, at $\sim 0.3 \mathrm{dex}$, to the $\mathrm{Na}$ 


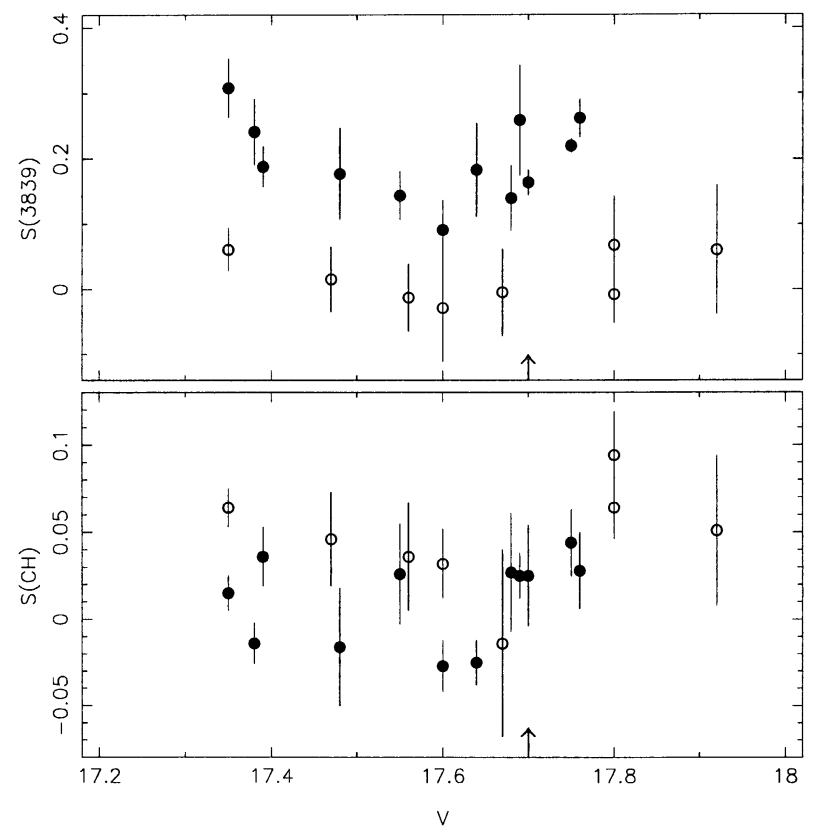

Figure 4. (a) Upper panel. Strength of the $\lambda 3883 \AA \mathrm{CN}$ band is plotted against $\mathrm{V}$ for stars near the main sequence turnoff in 47 Tuc. The main sequence turnoff is indicated by the arrow on the $\mathrm{x}$-axis. Stars can be unambiguously classified as CN-strong (filled symbols) or CN-weak (open symbols). (b) Lower panel. Strength of the G band (CH) at $\lambda \sim 4300 \AA$ is plotted against $\mathrm{V}$ for the same stars. In both panels the band strength increases upwards. Note the general tendency for CN-strong stars to be $\mathrm{CH}$-weak, and vice versa. All data from Briley et al. (1994).

abundance difference seen at much higher luminosities. This observation of $\mathrm{Na} / \mathrm{CN}$ line strength correlation at the main sequence turnoff presents two difficulties for the mixing hypothesis. First, it is extremely unlikely that the $\mathrm{NaNe}$ cycle operates to any significant extent in stars of this luminosity the interior temperatures are simply not high enough (even supposing the existence of a mixing mechanism to bring $\mathrm{Na}$ up to the surface layers which, as noted above, is unlikely). Second, the apparent agreement between the $\mathrm{Na}$ abundance difference at the main sequence turnoff and that near the red giant branch tip, strongly argues that no mixing driven processing can have occurred during the post-turnoff evolution. This contrasts markedly with the situation in the more metal-poor clusters where abundances are seen to change with evolutionary state.

Hence it seems likely that in 47 Tuc a primordial process is responsible for the abundance anomalies. If this is the case then we are presented with a dilemma: we can either claim that 47 Tuc is a rare or possibly unique cluster in that it possesses primordial abundance anomalies, or we must face the possibility that primordial processes play a significant role in gen- 
erating the $\mathrm{Na}$ abundance anomalies (at least) in other clusters. However, as noted originally by Norris et al. (1981) and Smith \& Norris (1982), the fundamental problem with the operation of a primordial process is not only to make it specific to the particular elements, here $\mathrm{C}, \mathrm{N}$ and $\mathrm{Na}$, but also to be able to generate the $\mathrm{C}$ depletions. In particular, in 47 Tuc the number of $\mathrm{CN}$-weak and $\mathrm{CN}$-strong stars are approximately equal, and they differ in C abundance by about a factor of two (e.g. Brown et al. 1990, Norris et al. 1984 ) with the $\mathrm{CN}$-strong (Na-strong) stars being $\mathrm{C}$ depleted. One is then forced to the requirement that if the $\mathrm{CN}$-strong stars form from a mixture of roughly equal amounts of original and processed/enriched material, then the processed/enriched material must contain only very small amounts of carbon. It is not at all clear that such a requirement can be met.

\section{Conclusions}

Despite intensive efforts on both the theoretical and observational fronts it is clear from the discussion above that we are far from a complete understanding of the origin(s) of abundance anomalies in globular cluster stars. So I will end this contribution with a list of goals that, if achieved, will allow considerable progress towards solving this very complex problem.

On the observational side, there are two questions (at least) that can be tackled. First, the observations of M13 show that $\mathrm{Mg}$ and $\mathrm{Al}$ abundance variations are still occurring at relatively faint luminosities $\left(\mathrm{M}_{\mathrm{V}} \approx+0.3\right)$. Nevertheless, in the context of the mixing hypothesis, there should be a luminosity on the RGB below which the $\mathrm{Mg}$ variations are no longer seen. This luminosity would correspond to the point in the evolution where the temperature in the vicinity of the H-burning shell first becomes hot enough to sustain significant ${ }^{24} \mathrm{Mg}$ to $\mathrm{Al}$ conversion. Second, it is very important to establish whether or not $\mathrm{Na}$ abundance variations are commonly seen on the main sequence in other clusters besides 47 Tuc. If such main sequence $\mathrm{Na}$ variations are common, then it will be very difficult to explain them within the evolutionary mixing hypothesis.

On the theoretical side there are also a number of issues that can be tackled. In terms of the mixing hypothesis, we need to resolve if any "simple" artificial mixing scheme can reproduce all the existing observational detail on the red giant branch. In other words, what modifications are required to reaction rates and internal temperature structure to reproduce the observations, and are such modifications plausible? Are we instead begining to see that "perturbations" of spherically symmetric 1D stellar models with mixing length convection are no longer adequate for this problem - a full 3D hydrodynamic calculation of giant branch evolution incorporating rotation and time dependent convection may be required instead! More fundamentally, we need further work on the physical mechanisms that could drive the mixing. But here it is especially important to keep in mind that the 
mechanism must be almost exclusively a globular cluster star phenomenon; field stars do not show these anomalies to anything like the same extent. As regards the primordial hypothesis, the fundamental requirement is to identify a process that is specific to the elements that are observed to vary, and to explain how such an enrichment (and depletion!) process can work in the context solely of globular cluster formation. Once again it is important to reproduce the observations quantitatively, not just qualitatively.

\section{References}

Arnould, M., Mowlavi, N., \& Champagne, A. 1995, in Stellar Evolution: What

Should Be Done, edited by A. Noels et al. (Univ. Liège, Liège), p. 17

Briley, M.M., Bell, R.A., Hoban, S., \& Dickens, R.J. 1990, ApJ, 359, 307

Briley, M.M., et al. 1994, AJ, 108, 2183

Briley, M.M., et al. 1996, Nature, 383, 604

Brown, J.A., \& Wallerstein, G. 1992, AJ, 104, 1818

Brown, J.A., Wallerstein, G., \& Oke, J.B. 1990, AJ, 100, 1561

Cavallo, R.M., Sweigart, A.V., \& Bell, R.A. 1996, ApJ, 464, L79

Cottrell, P.L., \& Da Costa, G.S. 1981, ApJ, 245, L79

Denisenkov, P., \& Denisenkova, S.N. 1990, Sov. Astron. Lett. 16, 275

Dickens, R.J., Croke, B.F.W., Cannon, R.D., \& Bell, R.A. 1991, Nature, 351, 212 Harding, G.A. 1962, Observatory, 82, 205

Hesser, J.E., Hartwick, F.D.A., \& McClure, R.D. 1976, ApJ, 207, L113

Kraft, R.P., et al. 1995, AJ, 109, 2586

Kraft, R.P., et al. 1997, AJ, 113, 279 (K97)

Langer, G.E., et al. 1986, PASP, 98, 473

Norris, J.E. \& Da Costa, G.S. 1995a, ApJ, 441, L81

Norris, J.E. \& Da Costa, G.S. 1995b, ApJ, 447, 680

Norris, J., \& Freeman, K.C. 1979, ApJ, 230, L179

Norris, J., \& Freeman, K.C. 1982, ApJ, 254, 143

Norris, J., Freeman, K.C., \& Da Costa, G.S. 1984, ApJ, 277, 615

Norris, J.E., Freeman, K.C., \& Mighell, K.J. 1996, ApJ, 462, 241

Norris, J., Freeman, K.C., Cottrell, P.L., \& Da Costa, G.S. 1981, ApJ, 244, 205

Osborn, W. 1971, Observatory, 91, 223

Paltoglou, G. 1989, PhD Thesis, Australian National University

Peterson, R.C. 1980, ApJ, 237, L87

Pilachowski, C.A., Sneden, C., Kraft, R.P., \& Langer, G.E. 1996, AJ, 112, 545

Popper, D.M. 1947, ApJ, 105, 204

Shetrone, M.D. 1996a, AJ, 112, 1517 (S96a)

Shetrone, M.D. 1996b, AJ, 112, 2639

Smith, G.H., \& Norris, J.E. 1982, ApJ, 254, 594

Smith, G.H., et al. 1996, AJ, 112, 1511

Suntzeff, N.B., \& Smith, V.V. 1991, ApJ, 381, 160

Vandenberg, D.A. \& Smith, G.H. 1988, PASP, 100, 314 